Methods: A registry retrospective descriptive study describing and exploring life-threatening situations leading to unexpected transfers to PICU in hospitalised children. The study includes all paediatric departments in the Central Denmark Region.

Perspective: This study will provide knowledge to assist the research efforts to identify and improve the management of critical ill children in paediatric wards.

\title{
PO03 - Experience from a group of adolescents with severe allergy asthma treatment with omalizumab
}

\author{
Anne Marie Bro Hofbauer (Denmark) ${ }^{1}$; Lone Agertoft (Denmark) ${ }^{1}$ \\ ${ }^{1}$ Hans Christian Andersen Children's Hospital. Odense University Hospital
}

Theme: Complex health care and chronic disease management.

Keywords: Adolescents, allergic asthma, education, omalizumab.

Background: Evidence supports that group educational interventions improve selfperception, self-confidence and attack management in asthma care.

Aim: To evaluate education and structured dialogue in enhancing knowledge and insight in the asthma disease, treatment with omalizumab and social behaviour.

Method: A group of four 13-16-year-olds received education using question and answer cards, and were informed about the effects and adverse effects of omalizumab. The dialogue included life before and after, relation to family and friends, ability and lack of ability to perform physical activities, and dreams of the future. One week later there was a telephone interview.

Results: All four adolescents experienced a positive change in everyday life and ability to manage the asthma disease. Furthermore, they all experienced an enhanced physical ability. In the interview they reflected on their own disease and physical ability.

Conclusion: Adolescents with a chronic disease benefit from sharing thoughts, emotions and knowledge about their disease and treatment.

\section{P004 - Metasynthesis approach in paediatric nursing literature: a scoping review}

Joana Romeiro (Portugal) ${ }^{1}$; Sílvia Caldeira (Portugal) ${ }^{1}$
${ }^{1}$ Instituto de Ciências da Saúde, Universidade Católica Portuguesa

Theme: Professional issues

Keywords: Child, family, metasynthesis, paediatric care, qualitative studies.

Introduction: Metasynthesis is defined as the synthesis of qualitative research. Despite the growing interest in this approach in adult nursing literature, it is not clear how it has been used in paediatric nursing literature. 